\title{
Homozygous Missense Mutation on Exon 22 of PKHD1 Gene Causing Fatal Autosomal Recessive Polycystic Kidney Disease
}

\author{
Sajina Sathyan ${ }^{1}$ Femitha Pournami ${ }^{1}$ Gopala Krishna Madhavilatha ${ }^{2}$ Amrit Tuteja ${ }^{1}$ \\ Anand Nandakumar ${ }^{1}$ Jyothi Prabhakar ${ }^{1}$ Naveen Jain ${ }^{1}$
}

${ }^{1}$ Department of Neonatology, Kerala Institute of Medical Sciences, Kerala, India

2 Medgenome Laboratories, Bengaluru, Karnataka, India

Address for correspondence Femitha Pournami, DM, Department of Neonatology, Kerala Institute of Medical Sciences, Trivandrum, Kerala, 695029, India (e-mail: femi_shifas@yahoo.com).

J Child Sci 2021;11:e70-e73.

\author{
Abstract \\ Keywords \\ - ARPKD \\ - PKHD1 \\ - polycystic kidney \\ disease \\ - fibrocystin- \\ polyductin complex
}

Autosomal recessive polycystic kidney disease, described as a congenital hepatorenal fibrocystic syndrome, is a significant inherited cause of end stage renal failure in children with reported incidence of 1 in 20,000 live births. The clinical spectrum is wide. Antenatal findings of echogenic reniform enlarged kidneys associated with evidence of intrauterine renal failure in the form of severe oligoamnios are pathognomonic. Postnatal illness ranges from fatal respiratory failure due to pulmonary hypoplasia in neonates to chronic kidney disease in children, or later presentation of ductal plate malformation and portal hypertension. Advances in genetic diagnostic techniques have allowed recognition of genotypes. We report a novel homozygous missense variant on exon 22 of PKHD1 gene (chr6:51915067G > A; c.2167C > T) that results in the amino acid substitution of cysteine for arginine at codon 723 (p.Arg723Cys). The affected neonate presented with antenatal anhydramnios, classical radiological features, and severe hypoxic respiratory failure likely due to pulmonary hypoplasia and succumbed. The parents were found to be heterozygous carriers. Detection of the specific variant in the proband facilitated prenatal investigation in the next pregnancy.

\section{Introduction}

Autosomal recessive polycystic kidney disease (ARPKD) is a rare disease with an incidence of approximately 1 in 20,000 live births. ${ }^{1}$ The carrier frequency in discrete populations is estimated to be 1 in $70 .^{2}$ Genetic confirmation in the proband could impact future pregnancies in the family and counseling.

The PKHD1 gene on chromosome 6p21.1-p12 that codes for fibrocystin/polyductin (FPC) is implicated primarily. ${ }^{3}$ FPC is localized to primary cilia and their basal bodies. The location, concentration, and presence during early ciliogenesis seem to indicate its role in microtubule organi-

received

October 20, 2020 accepted after revision January 26, 2021
DOI https://doi.org/ $10.1055 / \mathrm{s}-0041-1725175$. ISSN 2474-5871. zation and mechano-chemosensation (i.e., key functions of primary cilia). This in turn is vital to the maintenance of the structural integrity of various organs- notably renal and hepatic. ${ }^{4}$ Several mutations, mostly missense, have been reported. ${ }^{5}$ Most patients who have severe clinical features are compound heterozygotes. We report a rare missense homozygous mutation on exon 22 that presented perinatally. To the best of our knowledge, there are only three previous reports of this variant, in compound heterozygous form. Adding to the scientific literature may assist appropriate future pathogenicity classification of this unique variant and aid genetic counseling.

\section{(c) 2021. The Author(s).}

This is an open access article published by Thieme under the terms of the Creative Commons Attribution License, permitting unrestricted use, distribution, and reproduction so long as the original work is properly cited. (https://creativecommons.org/licenses/by/4.0/) Georg Thieme Verlag KG, Rüdigerstraße 14, 70469 Stuttgart, Germany 


\section{Patient Report}

Patient was referred to our institute at 31 weeks of gestation (nonconsanguineous union) with severe oligohydramnios and abnormal fetal kidneys on ultrasonography (USG). These findings were further classified as reniform enlargement of bilateral fetal kidneys (lengths of right and left kidneys were 8.6 and $6.5 \mathrm{~cm}$, respectively), diffuse hyperechogenecity, poor corticomedullary differentiation (CMD), and no urine in fetal bladder or discernible liquor pockets. The fetal liver was unremarkable. The family received a provisional diagnosis of ARPKD. The baby was delivered through anhydramnios at 35 weeks of gestation. The neonate, who was critically ill from birth, had features of hypoxic respiratory failure and required high-frequency oscillatory ventilation. A clinical diagnosis of pulmonary hypoplasia due to ARPKD was supported by the rapidly deteriorating clinical course, occurrence of tension pneumothorax, and anuria (-Fig. 1). USG showed enlarged reniform kidneys, of sizes $9.3 \times 4.6 \mathrm{~cm}$ (right) and $9.3 \times 3.3 \mathrm{~cm}$ (left), with multiple tiny cysts, altered CMD, no renal pelvis/ureteral dilatation, and no urine in bladder. USG of liver did not reveal any abnormality. The baby succumbed on day 2 of life. The family was counseled by the senior genetic counselor and they sought testing for cystic diseases of kidney.

The sequences obtained after next generation sequencing (NGS) were aligned to human reference genome (GRCh37/ hg19) by using BWA program ${ }^{6,7}$ and analyzed by using Picard and GATK version 3.6 to identify variants relevant to the clinical indication. ${ }^{8,9}$ GATK best practices framework for the identification of variants in the sample were followed. Clinically relevant mutations were annotated by using published variants in the literature and a set of disease databases ClinVar, OMIM, GWAS, HGMD, and SwissVar. ${ }^{10-14}$ Common variants were filtered based on allele frequency in 1000 Genome Phase 3, ExAC, EVS, dbSNP147, 1000 Japanese Genome, and MedGenome Laboratory's internal Indian population database. ${ }^{15,16}$ Nonsynonymous variants effect was

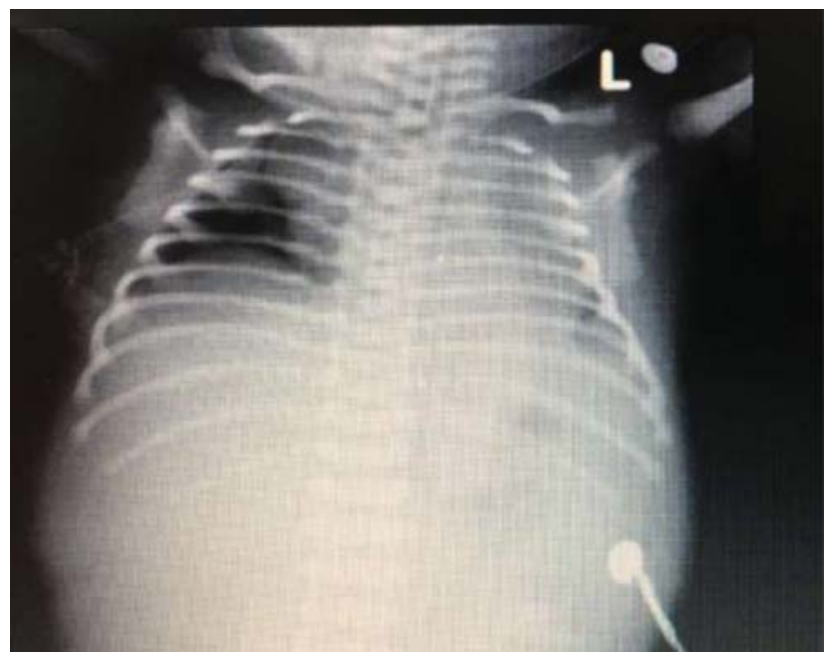

Fig. 1 X-ray of chest and abdomen; right pneumothorax, low volume lung, abdomen almost completely gas less (due to enlarged kidneys filling the abdomen). calculated by using multiple algorithms such as PolyPhen-2, sorting intolerant from tolerant (SIFT), Mutation Taster2, Mutation Assessor, and likelihood ratio test (LRT). A homozygous missense variation in exon 22 of the PKHD1 gene (chr6:51915067G > A; c.2167C $>$ T) that results in the amino acid substitution of cysteine for arginine at codon 723 (p. Arg723Cys) was identified in the index patient, which was further validated by Sanger sequencing. A same variant affecting the same codon has previously been reported as one of the compound heterozygous variants in a patient affected with autosomal recessive polycystic kidney disease. ${ }^{17}$ The in silico prediction of the variant is benign by SIFT, LRT, and MutationTaster2. The reference codon is conserved across mammals. The variant was classified as variant of uncertain significance (VUS).

The same variant was detected in the heterozygous state in both parents on Sanger sequencing. The kidneys and liver of both parents were screened by USG and found to be normal, indicating that they were asymptomatic carriers. The family visited with their second pregnancy 2 years later. After the couple was counseled regarding prenatal testing of a VUS, they decided to go for amniocentesis. The fetus was found to be heterozygous for the variant identified in the index child. Follow-up scans were normal and a healthy female baby was delivered at term. Her postnatal USG was reported normal and she remains well on follow-up. Sequence chromatograms of the parents and second fetus are represented in - Fig. 2.

\section{Discussion}

The suspicion of ARPKD is based on clinical and radiological findings in the proband with normal renal status in both parents. Presence of typical radiological findings (i.e., antenatally or postnatally) with evidence of hepatic involvement, normal parental renal status, and genetic/pathological confirmation is required to classify as ARPKD. ${ }^{18}$ Renal disease may present in neonates as nephromegaly, hypertension, and renal dysfunction. The majority of patients progress rapidly to end stage renal disease requiring renal replacement therapy. Sick neonates have features of pulmonary hypoplasia, and approximately $30 \%$ die in infancy. ${ }^{19}$

As advances in renal replacement therapy and kidney transplantation improve long-term survival, it is likely that clinical hepatobiliary disease will become a major feature of the natural history of ARPKD. In addition, a subset of affected individuals are identified later with hepatosplenomegaly; in whom the renal disease is often mild and may be discovered incidentally during imaging studies of the abdomen. In our patient, typical clinical and radiological presentation left little doubt about the diagnosis. Presentation as liver disease may be the case in older children. ${ }^{20}$ Some patients develop histologic liver involvement, which is reflected by the disease term "polycystic kidney and hepatic disease 1 (PKHD1)". Defective remodeling of the ductal plate leads to biliary atresia, and hepatic fibrosis that can result in complications such as hypersplenism and esophageal varices. 
e72 New Variant Causing Autosomal Recessive Polycystic Kidney Disease Sathyan et al.
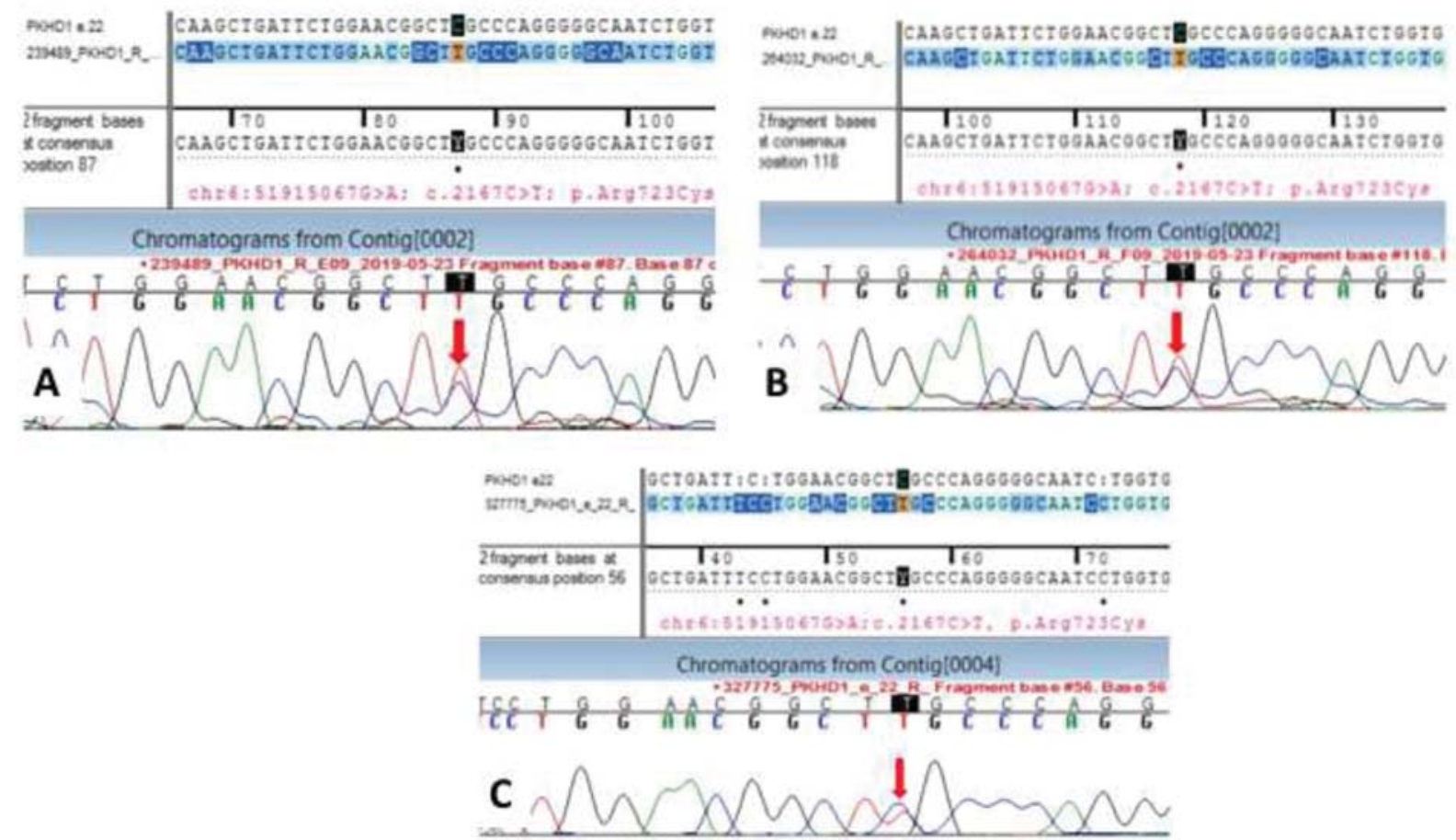

Fig. 2 Sequence chromatograms and alignment to the reference sequence showing the variation in exon 22 of the PKHD1 gene (chr6:51915067G > A; c.2167C > T; p.Arg723Cys) detected in heterozygous condition. (A) Mother, (B) father, (C) and fetus of second pregnancy (sibling-to-be) of the index patient.

Discovery of the genetic basis of the disease as PKHD1 gene mutations in the 1950s was a milestone. The principal role of the coded protein, FPC seems to be localized to the primary cilia and basal body and might be involved in microtubule organization. ${ }^{4}$ The protein is considered to undergo post-translational modifications, but the function is not entirely known. Mutations implicated in ARPKD are evenly distributed along the length of the PKHD1 gene. Therefore, it is hypothesized that a certain amount of fulllength functional protein is required for function. Another proposed mechanism is that mutations disrupt the relative abundance of functional isoforms of FPC, which disturb their required stoichiometric ratios. ${ }^{2}$

Presence of two truncating mutations is associated with severe clinical presentation during the neonatal period. Some missense mutations can, however, be as severe as truncating mutations. This may be related to as yet unidentified modifying genes (including those in noncoding intronic regions), complex transcriptional profiles, and epigenetic influences. Recently, mutations in DZIP1L (which codes for ciliary TZ protein) have been described. These patients usually follow a moderate clinical course. ${ }^{2}$

In our case, a homozygous missense variation was identified in exon 22 . This variant has been previously reported as one of the compound heterozygous variants in a patient affected with autosomal recessive polycystic kidney disease. ${ }^{21}$ Szabo et al ${ }^{17}$ reported a neonate who presented with respiratory failure with compound heterozygous variants on exon 22 c. $2167 \mathrm{C}>\mathrm{T}$ and exon 58 c.8870 $\mathrm{T}>\mathrm{C}$. Gunay-Aygun et $\mathrm{al}^{5}$ included survivors beyond 6 months of age and identified 77 PKHD1 variants, they found one
Caucasian nonperinatal patient with compound heterozygous variant in exon 22 c.2167C $>$ T and c.34669C $>$ G.

Deleterious effects of any variation consist of damage to protein structures and gene regulation. Alterations in the protein sequence may ultimately affect protein charge, geometry, hydrophobicity, dynamics, translation, or inter/intraprotein interaction. In silico prediction algorithms use this information to predict the damaging effect of a particular variation. This variant "c.2167C $>$ T" in exon 22 of PKHD1 gene has been classified as benign by SIFT, Polyphen-2 and Mutation Taster2. Gunay-Aygun et al also classified it to be probably benign. We have observed this variant to be phenotypically associated with typical clinical and radiological presentation in homozygous state in the deceased proband in our case. Segregation in the parents, and the carrier status in the healthy younger sibling has been confirmed by Sanger sequencing. The previous reports and our case indicate that this variant is probably damaging and may be associated with the disease. When more case reports and/or experimental evidences based on targeted mutagenesis become available in the future, it is possible that the classification of this variant may change.

\section{Conclusion}

Homozygous missense variation on exon 22 of PKHD1 gene described here was associated with typical features of perinatal ARPKD. This is a case report that adds to the disease database.

Funding

None. 


\section{Conflict of Interest}

None declared.

\section{References}

1 Hartung EA, Guay-Woodford LM. Autosomal recessive polycystic kidney disease: a hepatorenal fibrocystic disorder with pleiotropic effects. Pediatrics 2014;134(03):e833-e845

2 Bergmann C. Genetics of autosomal recessive polycystic kidney disease and its differential diagnoses. Front Pediatr 2018;5:221

3 Al-Bhalal L, Akhtar M. Molecular basis of autosomal recessive polycystic kidney disease (ARPKD). Adv Anat Pathol 2008;15(01): 54-58

$4 \mathrm{Kim}$ I, Fu Y, Hui K, et al. Fibrocystin/polyductin modulates renal tubular formation by regulating polycystin-2 expression and function. J Am Soc Nephrol 2008;19(03):455-468

5 Gunay-Aygun M, Tuchman M, Font-Montgomery E, et al. PKHD1 sequence variations in 78 children and adults with autosomal recessive polycystic kidney disease and congenital hepatic fibrosis. Mol Genet Metab 2010a99(02):160-173

6 LiH, Durbin R. Fast and accurate long-read alignment with BurrowsWheeler transform. Bioinformatics 2010;26(05):589-595

7 Meyer LR, Zweig AS, Hinrichs AS, et al. The UCSC Genome Browser database: extensions and updates 2013. Nucleic Acids Res 2013; 41(Database issue, D1):D64-D69

8 McKenna A, Hanna M, Banks E, et al. The genome analysis toolkit: a MapReduce framework for analyzing next-generation DNA sequencing data. Genome Res 2010;20(09):1297-1303

9 Li H, Handsaker B, Wysoker A, et al; 1000 Genome Project Data Processing Subgroup. The sequence alignment/map format and SAMtools. Bioinformatics 2009;25(16):2078-2079

10 Landrum MJ, et al. ClinVar: public archive of interpretations of clinically relevant variants. Nucleic Acids Res 2015
11 Hamosh A, Scott AF, Amberger JS, Bocchini CA, McKusick VA. Online mendelian inheritance in man (OMIM), a knowledgebase of human genes and genetic disorders. Nucleic Acids Res 2005;33 (Database issue):D514-D517

12 Welter D, MacArthur J, Morales J, et al. The NHGRI GWAS Catalog, a curated resource of SNP-trait associations. Nucleic Acids Res 2014;42(Database issue):D1001-D1006

13 Stenson PD, Ball EV, Mort M, et al. Human gene mutation database (HGMD): 2003 update. Hum Mutat 2003;21(06):577-581

14 Mottaz A, David FP, Veuthey AL, Yip YL. Easy retrieval of single amino-acid polymorphisms and phenotype information using SwissVar. Bioinformatics 2010;26(06):851-852

15 Auton A, Brooks LD, Durbin RM, et al; 1000 Genomes Project Consortium. A global reference for human genetic variation. Nature 2015;526(7571):68-74

16 Analysis of protein-coding genetic variation in 60,706 humans. Nature 2015;536(7616):285-291

17 Szabó T, Orosz P, Balogh E, et al. Comprehensive genetic testing in children with a clinical diagnosis of ARPKD identifies phenocopies. Pediatr Nephrol 2018;33(10):1713-1721

18 Sweeney WE, Avner ED. Polycystic Kidney Disease, Autosomal Recessive 2001 Jul 19. In: Adam MP, Ardinger HH, Pagon RA, et al., eds. GeneReviews ${ }^{\circledR}$ [Internet]. Seattle, WA: University of Washington; 1993-2020. Available at: https://www.ncbi.nlm.nih.gov/books/NBK1326/

19 Hoyer PF. Clinical manifestations of autosomal recessive polycystic kidney disease. Curr Opin Pediatr 2015;27(02):186-192

20 Shneider BL, Magid MS. Liver disease in autosomal recessive polycystic kidney disease. Pediatr Transplant 2005;9(05): 634-639

21 Publications on cystic kidney disease of our group. Accessed October 4, 2020 at: http://www.humgen.rwth-aachen.de/index. php?page=publications 